\title{
Femtocell Collaborative Outage Detection (FCOD) with Built-in Sleeping Mode Recovery (SMR) Technique
}

\author{
Dalia Abouelmaati $\uparrow$, Arsalan Saeed $\dagger$, Oluwakayode Onireti $\uparrow$, Muhammad Ali \\ Imran $\dagger$, Kamran Arshad \\ $\dagger$ Institute for Communication Systems (ICS), University of Surrey, Guildford, UK. \\ †Department of Engineering Systems, University of Greenwich, Kent, United Kingdom \\ d.y42011@gmail.com, \{arsalan.saeed, o.s.onireti, \\ m.imran\}@surrey.ac.uk, k.arshadegre.ac.uk
}

\begin{abstract}
Self-Organizing Networks (SONs) have an important role in the development of the next generation mobile networks by introducing automated schemes. Cell outage detection is one of the main functionalities in self-healing mechanism. Outage detection for small cells has not been discussed in literature with greater emphasis yet. The Femtocell Collaborative Outage Detection (FCOD) algorithm with built-in Sleeping Mode Recovery (SMR) is introduced in this paper. The proposed algorithm is mainly based on the femtocell collaborative detection with incorporated sniffer. It compares the current Femtocell Access Points FAPs' Reference Signal Received Power (RSRP) statistics with a benchmark data. An outage decision is autonomously taken by each FAP depending on a certain threshold value. Moreover, the FCOD algorithm is capable of differentiating between the outage and sleeping cells due to the presence of the built-in SMR technique.
\end{abstract}

Keywords: Self-organizing networks, self-healing, cell outage detection, heterogeneous cellular network.

\section{Introduction}

Self-Organizing Networks (SONs) have lately been a captivating paradigm for the next-generation cellular networks via standardization bodies [1]-[3]. The aim of SON is to introduce autonomic features such as self-configuration, self-optimization and self-healing functionalities. SON functionalities will therefore enable the automation of certain activities performed by the network operator, thus leading to lower operating expenditure, simplified management and improved efficiency [4]. Selfhealing involves automated remote detection of faults and recovery processes to compensate the faults in the network. Cell outage detection is considered as an important stage in the self-healing functionality. The basic function of the detection phase is to automatically detect the cells in outage, i.e. the cells that cannot offer services due to software failures, environmental disasters, technical fault, or component malfunctions [3]. Cell outage causes coverage and capacity gaps, which lead to high user churn rate, as well as increased operational costs [5]. In some cases, 
cell outage can easily be detected by the Operations and Support System (OSS), while some detection might require unplanned site visits, which is a costly task [5], [6].

Cell outage detection algorithms proposed in [7]-[9] are focused on macro-cells. It is expected that future cellular networks will be heterogeneous networks (HetNets), i.e., a mix of macro-cells for ubiquitous user experience and small cells or femto access points (FAPs) for high data rate transmission. Hence, the algorithms proposed in [7]-[9] are not suitable for such networks due to the dense deployment nature of FAPs in the HetNets, as compared to the macro only deployments. Furthermore, there is high possibility of having sparse user statistics in small cells, since they usually support very few users as compared to macro-cells. Recently, [1] proposed a Collaborative Outage Detection (COD) scheme, which is based on the implementation of a distributed outage trigger mechanism and sequential hypothesis testing within a predefined cooperation range. This scheme depends mainly on the Reference Signal Received Power (RSRP) statistics of the users within the cooperative range. Consequently, this approach will fail in detecting cell outage if there are no active users within the cooperation range. Furthermore, the COD and the conventional cell outage detection schemes in literature do not consider sleep mode of FAPs. Therefore, a FAP in idle/sleep mode will be mistakenly taken as in outage, which results in unnecessary compensation procedures and extra costs.

As a solution to the aforementioned challenges, energy efficient Femtocell Collaborative Outage Detection (FCOD) with a built-in Sleeping Mode Recovery (SMR) algorithm is proposed to automatically detect cell outage, by using performance statistics analysis of the collaborative FAPs. The FCOD technique is able to detect cell outage, even in the absence of users and scenarios with low FAPs density within the collaborative range. We consider the energy efficient node controlled mode for the FAPs sleep/wakeup mode. This self-controlled process requires a sniffer and a micro controller to be added to the FAPs to control the sleep and the wakeup cycles [10]. The rest of this paper is organized as follows: In Section II, we present the network model. In Section III, we present our proposed FCOD algorithm with SMR. In Section IV, we present extensive simulation based results to substantiate the performance of our proposed algorithm. Finally, we draw the conclusions in Section V.

\section{Network Model}

We consider a typical heterogeneous network (HetNet) with $F A P s \mathcal{F}=\{1, \ldots, F\}$ overlaid on a macrocell. We also consider that one of the femtocells suffers an outage with certain probability in the operational process. The FAP in outage is not able transmit or receive any signal. Furthermore, another femtocell is switched into the sleep mode. The locations of FAPs are assumed to be known to the macrocell base station (MBS). The FAPs transmission powers are assumed to be constant through the outage detection process. In the downlink, FAPs are periodically transmitting reference signals, which assist the channel measurements of the user i.e., RSRP measurement. These measurements are reported to the FAPs as feedback messages, which help to decide whether there is an outage or not. 
We consider that users' positions are unknown to the FAPs and MBS. The users periodically report the neighboring FAPs' RSRP statistics periodically to their serving FAPs, which is used in handover decision and cell reselection process. We assume that the users in a certain area $A$ follow a Poisson point process, $n_{A} \sim \operatorname{Poi}(n ; \rho|A|)$, where $\rho$ is the density and $n_{A}$ is the number of users within a certain area $A$.

The channel gains of a user $u$ to a FAP $f$ are expressed based on the model described in [11] as:

$$
h=\left(\frac{d_{o}}{d_{u, f}}\right)^{a} e^{X_{u, f}} e^{Y u, f},
$$

where $d_{o}$ is the reference distance $(1 \mathrm{~m}), d_{u, f}$ the distance between FAP $f$ and user $u$, $a$ is the path loss exponent, while $e^{X_{u, f}}$, and $e^{Y} u, f$ are the shadowing fading factor and multi-path fading factor, respectively. The shadowing fading follows a Gaussian distribution defined by $X_{u, f} \sim N(0, \sigma), \forall u, f$. The multipath fading is Rayleigh fading with zero mean, and therefore $E\left[e^{Y u, f}\right]=0$. We assume that the effects of shadowing-fading are independent over time. According to this hypothesis, the user's RSRP statistics are independent random variables. All the RSRP statistics can be described using (1). Therefore, this distribution can be described according to [12] as follows:

$$
r_{u} \sim\left\{\begin{array}{cc}
N\left(N_{o}, \frac{N_{o}^{2}}{M}\right) & H_{o} \\
N\left(P_{u}+N_{o}, \frac{\left(P_{u}+N_{o}\right)^{2}}{M}\right) & H_{1}
\end{array}\right.
$$

where $r_{u}$ is the RSRP statistics for user $u, P_{u}$ is the received signal strength for user $u, N_{o}$ is the noise power, $M$ is the number of samples of the signal $\left(1.4 \times 10^{3} / \mathrm{ms}\right.$ for 1.4 $\mathrm{MHz}$ bandwidth). $H_{0}$ and $H_{1}$ denote the outage and normal hypothesis.

\section{FCOD-SMR ALGORITHM}

\subsection{FCOD with Trigger Stage}

Sleep Mode Recovery (SMR) Technique is introduced in this paper to prevent the sleeping FAP from been mistaken as in outage. When a FAP wants to switch to the sleeping mode, it informs the other FAPs within the collaborative range. These FAPs will then replace the current statistics of the sleeping FAP with the benchmark data for this FAP, which represent the normal state, before they start sensing the outage. 


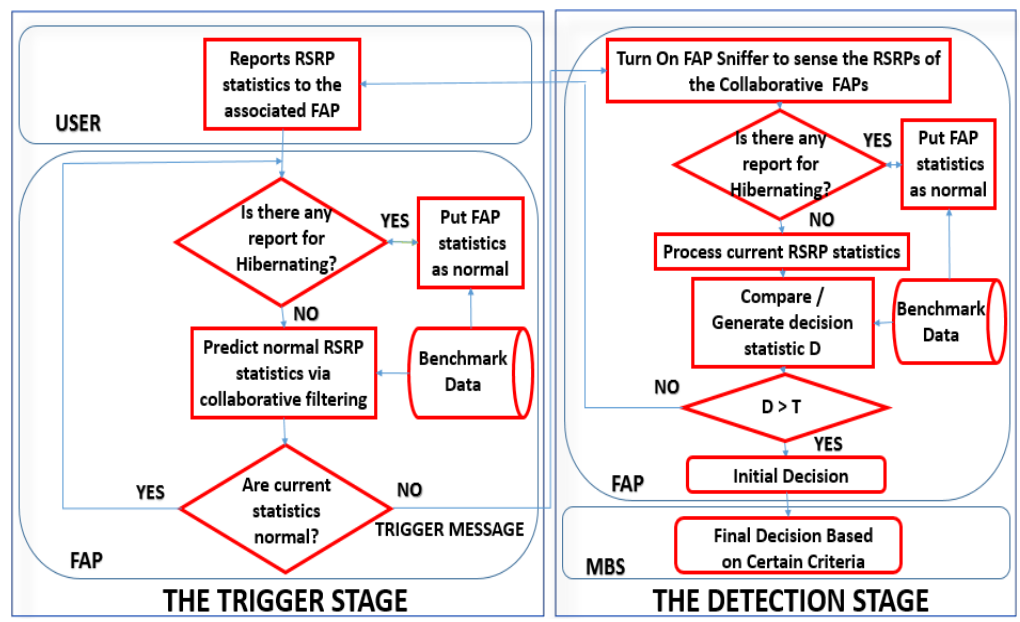

Fig. 1. FCOD Algorithm

When the sleeping FAP becomes active, it informs the FAPs within its collaborative range, in order to be treated as normal. Fig. 1 shows the flowchart for the FCOD algorithm, which includes the SMR approach. The FCOD algorithm involves two stages: the trigger stage and the detection stage.

The energy efficient FCOD technique is based on the node controlled mode, where the FAP detecting outage (i.e. FAP 4 in Fig. 2) uses a sniffer and a micro controller to sense UE activity in order to switch between the sleeping and wakeup mode. When the FAP senses UE activity, it wakes up only if the sensed UE is its subscriber, this avoids the unnecessary activation of the FAP in case of presence of a non-subscriber UE in the vicinity [10]. Once no authorized UE activity is detected the SMR approach will be initiated, FAP 4 will inform the rest of the neighboring FAPs (i.e. FAP 2 and FAP 3) and users within the collaborative range before it switches to the sleep mode as shown in Fig. 2. The collaborative FAPs (i.e. FAP 2 and FAP 3) and users will use the benchmark data (database of normal RSRP statistics) to replace the current RSRP statistics for the sleeping FAP (i.e. FAP 4). Consequently, the sleeping FAPs will not be falsely detected as in outage. After the sleeping FAP becomes active again, it informs the collaborative FAPs in order to be treated normally.

The benchmark data is frequently updated in case new FAPs are introduced into the network or any other changes occur within the collaborative range. The trigger stage, which includes the SMR, is used to check any abnormality (usually an outage) in the FAPs by using the reported user's RSRP statistics to trigger the detection stage. Consequently, the sniffer is not kept on all the time. In the detection stage each FAP within a certain collaborative range $(\mathrm{R})$ uses a sniffer (such as the one used in the node controlled mode to sense the UE activity but with different sensitivity) to sense the neighboring FAPs' current RSRP statistics within a certain collaborative range (the range will be determine according to the sensitivity of the sniffer). 


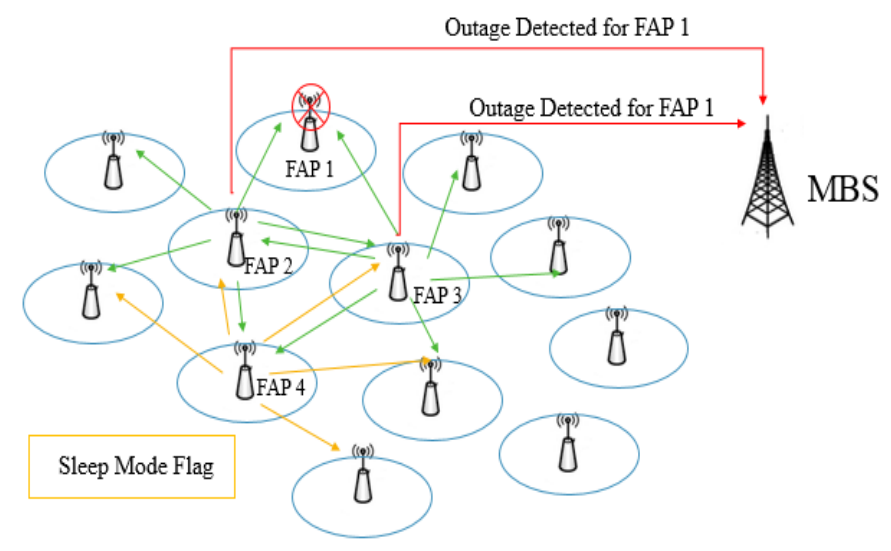

Fig. 2. Conceptual Model for FCOD

The current RSRPs statistics are compared to the benchmark data, which is the previously stored FAPs' RSRP statistics in the normal state. This benchmark data can be stored and exchanged between a group of collaborative FAPs. If the decision statistic $(D)$ for a certain FAP is greater than a certain Threshold value $(T)$, this FAP (i.e. FAP 1) will be initially decided as in outage. The rest of the FAPs within the collaborative range will start sensing using their sniffers. Centralized synchronization is used to manage the initiation of detection for the collaborative FAPs. The initial decision for an outage will be reported to the MBS. The detection stage will always be able to detect the outage regardless of the number of users within the collaborative range. $D$ is determined as follows:

$$
D=R S R P-R S R P_{0}
$$

where $R S R P$ is the normal RSRP statistics from the benchmark data and $R S R P_{0}$ is the sensed current RSRP statistic for a certain collaborative FAP.

The outage decision is based on the following equation:

$$
D>T \text {, }
$$

where $T$ is a heuristically predefined threshold, which is dependent on the false alarm and misdetection rates.

The MBS will check the initial decision reported from the collaborative FAPs (FAP 2 and FAP 3) as shown in Fig. 2. If more than 5\% of the FAPs within a certain collaborative range reported an initial decision of an outage for the same FAP, then the MBS will take the final decision that this FAP is in outage. Subsequently, the MBS will start the necessary outage compensation scheme.

The FAPs might need to sense the collaborative users from time to time, in order to avoid delay or undiscovered outage in the trigger stage in case there is no user in the collaborative range. If there are no users sensed, the detection stage will be triggered immediately without waiting for the trigger stage. Another solution for the absence of users is that the detection stage might be triggered randomly from time to time or at a regular time interval. 


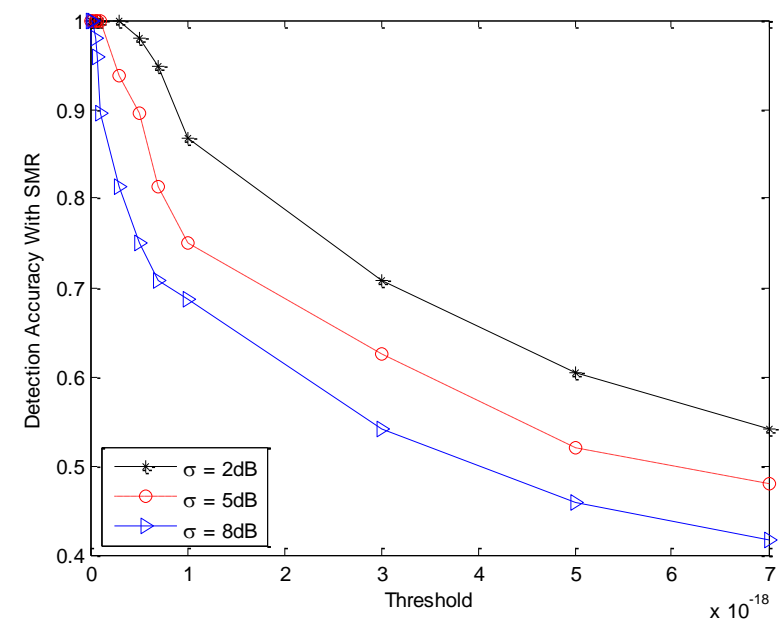

Fig. 3. Detection Accuracy with SMR versus the Threshold for FCOD

\subsection{FCOD without Trigger Stage}

This scenario is similar to the previous scenario but it only includes the detection stage. The trigger stage is replaced by a timer, to initiate the detection stage in regular time intervals. This eliminates the overhead caused by the trigger stage. However, this scheme might increase the outage detection delay, especially if the outage occurs just after the detection stage, which means the outage won't be detected until the next detection interval.

\section{Simulation Results}

Simulation Scenario: We consider a two-tier cellular system, which contains multiple femtocells within a macrocell. Femtocells are randomly distributed within the macrocell area (with radius $\mathrm{r}=1000 \mathrm{~m}$ ). We assume that FAPs transmit with fixed power and the carrier frequency is $2.5 \mathrm{GHz}$ with channel bandwidth of $1.4 \mathrm{MHz}$. The users of the femtocell are randomly distributed within the femtocell area (with radius $\mathrm{r}=50 \mathrm{~m}$ ). Furthermore, the users are connected with the FAP with the strongest RSRP. The path loss exponent a is set to 4. The number of FAPs and users will vary according to the different scenarios considered. However, the maximum number of FAPs used is 49 and the minimum number of users is 1 . The transmission power of the $\mathrm{FAP}$ Po $=5 \mathrm{dBm}$, maximum cooperative range considered $\mathrm{R}=600 \mathrm{~m}$, and the shadow fading standard deviation $\sigma \mathrm{dB}=8 \mathrm{~dB}$. The FCOD algorithm does not have any restriction on the parametric values (number of users or FAPs).

Fig. 3 illustrates the performance of the FCOD algorithm with SMR. It shows the detection accuracy versus the heuristically set threshold. Furthermore, it illustrates that by using small threshold values, the accuracy is improved significantly. The reason is that if the difference between the normal RSRP statistics from the 




Fig. 4. False Detection versus Threshold for FCOD

benchmark data and the current RSRP is not large, it will be able to detect the outage. Moreover, the figure also shows that when shadowing fading increases $(\sigma=8)$, the detection stage becomes less accurate than in case of less shadowing $(\sigma=2)$. This is because of the errors introduced by the shadow fading.

Another algorithm is developed to evaluate the false detection with several threshold values in different channel conditions. Fig. 4 shows the performance of the FCOD algorithm with SMR. It shows the false detection (due to choosing inappropriate threshold value) versus the threshold. Furthermore, it illustrates that by using higher threshold values, the false detection rate increased significantly. The reason is that if the difference between the normal RSRP statistics and the current RSRP is not large, it won't be able to detect the outage. Moreover, the figure also shows that when shadowing fading increases $(\sigma=8)$, the false detection rate increases during the detection stage compared to the less shadowing $(\sigma=2)$ case. This is because of the errors introduced by the shadowing fading. Due to the significant importance of differentiating between the outage case and the sleeping mode case, the SMR technique is introduced in this paper to avoid the false detection of the sleeping FAP as an outage. It's also crucial to presents the false detection due to the absence of SMR with several threshold values in different channel conditions.

Fig. 5 demonstrates the performance of the FCOD algorithm without SMR. It shows the false detection versus the threshold. Furthermore, it illustrates that by using small threshold values, the false detection rate increased significantly. As the detection (either false or correct detection) is better with smaller threshold values. The reason is that if the difference between the normal RSRP statics and the current RSRP is not large, it will still be able to detect the outage. However, in this case it's a false detection as it is a sleeping FAP not outage FAP. Moreover, Fig. 5 also shows that when shadowing fading increases $(\sigma=8)$, the false detection rate decreases compared to the case of less shadowing $(\sigma=2)$. This is because of the errors introduced by the shadowing fading, which affects the false detection.

Fig. 6 illustrates the performance of the FCOD algorithm with SMR. It shows the detection delay versus FAP transmission power. Furthermore, it shows that the 




Fig. 5. False Detection-FCOD without SMR versus Threshold

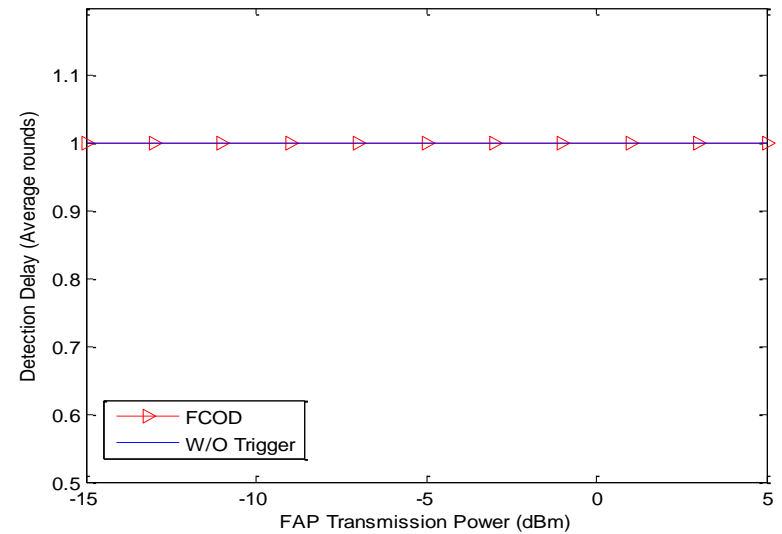

Fig. 6. Detection Delay versus FAP Transmission Power for FCOD

average delay for the FCOD with and without the trigger stage is one round, which means that the trigger stage doesn't affect the delay of the FCOD. The trigger stage function is to optimize the use of the sniffer by not keeping it on all the time. If the trigger stage senses an abnormality of a certain FAP it will initiate the detection stage by turning on the sniffer of the sensing FAP. However, the trigger stage increases the overhead of the FCOD algorithm.

Fig. 7 illustrates the performance of the FCOD algorithm with SMR. It shows the detection accuracy versus FAP transmission power with different threshold values. Furthermore, it demonstrates that by using a lower threshold value it is possible to achieve $100 \%$ accuracy without increasing the FAP transmission power. However, 


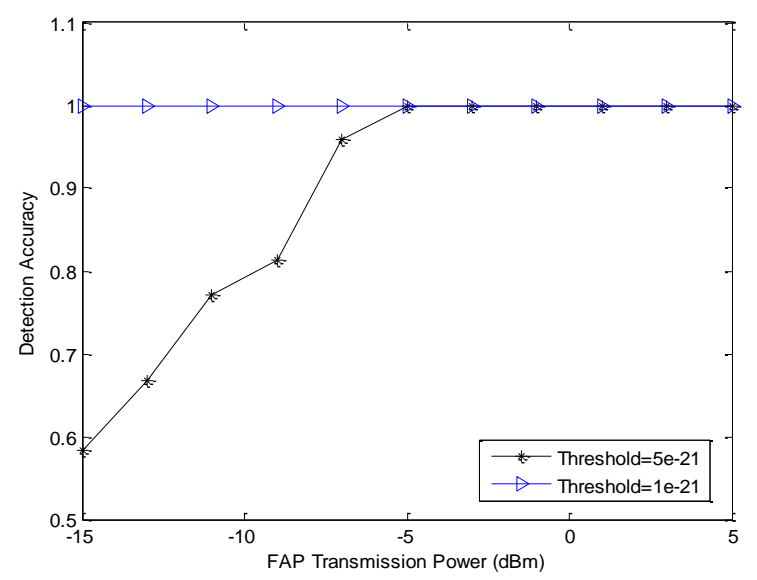

Fig. 7. Detection Accuracy versus FAP Transmission Power

when a higher threshold value is required due to a limitation of channel condition (power variation will set a limitation for using lower threshold as it might be misleading), a higher accuracy is still be achievable but at a cost of higher transmission power.

Fig. 8 illustrates the performance of the FCOD algorithm with and without SMR. It represents the percentage of FAPs sensing the outage versus the cooperative range, for the case of false and correct detection. Furthermore, it demonstrates that in case of using SMR technique when the collaborative range increases the percentage of FAPs sensing the outage increases, which improve the reliability of the FCOD algorithm. However this collaborative range is limited by the sniffer sensitivity. This figure can be used by the MBS to decide on an outage FAP based on a certain criteria. For example, if the collaborative range $\mathrm{R}=600 \mathrm{~m}, 100 \%$ of the FAPs should detect the outage. The MBS then decide (according to the criteria) that if 5\% of these FAPs report an outage for a certain FAP, a final decision about the outage will be taken by the MBS. Fig. 8 also shows the case of false detection without the use of SMR technique. In this case nearly half the percentage of the FAPs falsely detected the sleeping FAP as an outage FAP. Consequently, more costs will be required to compensate the false outage.

\section{Conclusion}

The energy efficient FCOD algorithm with a high level of accuracy in detecting FAP outage is introduced. The proposed algorithm offers significant reduction in the communication overhead and the detection delay for the adopted two-tier macrofemto scenario. Also it is capable of detecting the outage with minimum users or FAPs density within the collaborative range. Furthermore, the FCOD algorithm is able to differentiate between cell outage and sleeping cells with the aid of the SMR technique. 


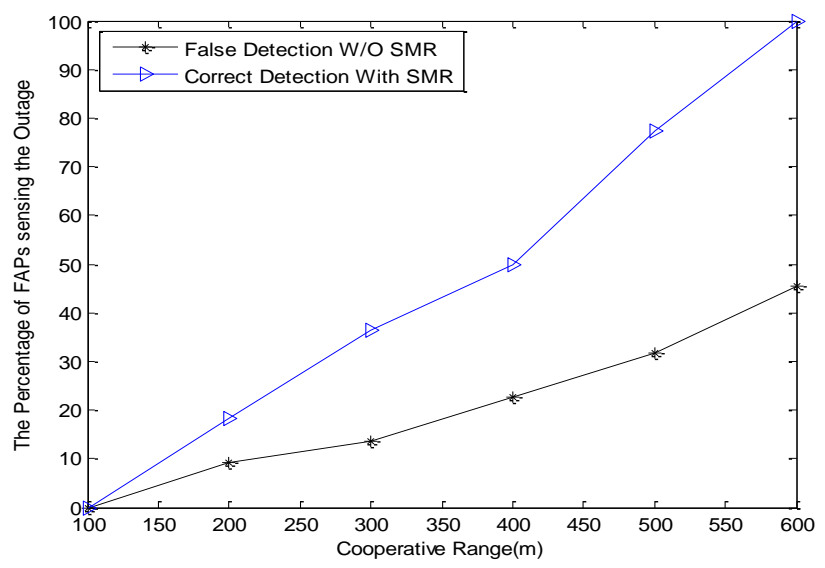

Fig. 8. The Percentage of FAPs Sensing the Outage versus Cooperative Range

Acknowledgments. This research leading to these results was partially derived from the University of Greenwich Research \& Enterprise Investment Programme grant under agreement number RAE-ES-01/14.

\section{References}

1. Wang, W., Zhang, J., Zhang, Q.: Cooperative Cell Outage Detection in Self-Organizing Femtocell Networks. In: IEEE INFOCOM (2013)

2. Telecommunication Management; Self-organizing Networks (SON) Policy Network Resource Model (NRM) Integration Reference Point (IRP); Information Service (IS). In: 3GPP TS 32.522, Rel. 9 (2010)

3. Telecommunication Management; Self-organizing Networks (SON); Self-healing Concepts and Requirements. In: 3GPP TS 36.902, Rel.10 (2010)

4. Combes, R., Altman, Z., Altman, E.: Self-organization in Wireless Networks: A Flow-level Perspective. In: IEEE INFOCOM (2012)

5. Amirijoo, M. et al.: Cell Outage Management in LTE Networks. In: COST 2100 TD (09)941 (2009)

6. Self-organizing Networks, NEC's Proposals for Next-Generalization Radio Network Management. In: NEC White Paper (2009)

7. Zoha, A., Saeed, A., Imran, A., Imran, M. A., Abu-Dayya, A.: A SON Solution for Sleeping Cell Detection using Low-Dimensional Embedding of MDT Measurements. In IEEE International Symposium on Personal, Indoor and Mobile Radio Communications (2014)

8. Liao, Q., Wiczanowski, M., Stanczak, S.: Toward Cell Outage Detection with Composite Hypothesis Testing. In: IEEE International Conference on Communications (2012)

9. Khanafer, R. Solana, B., Triola, J., Barco, R., Moltsen, L., Altman, Z., L'azaro, P.: Automated Diagnosis for UMTS Networks Using Bayesian Network Approach. In: IEEE Trans. Veh. Technol., vol. 57, no. 4 (2008) 
10. Navaratnarajah, S., Saeed, A., Dianati, M., Imran, M.: Energy Efficiency in Heterogeneous Wireless Access Networks. In: IEEE Wireless Communications Magazine, vol. 20, no.5 (2013)

11. Erceg. V. et al.: An Empirically Based Path Loss Model for Wireless Channels in Suburban Environments. In: IEEE Journal on Selected Areas in Communications, vol. 17, no. 7 (1999)

12. Shellhammer, S. et al.: Performance of Power Detector Sensors of DTV Signals in IEEE 802.22 WRANs. In Proc. ACM TAPAS (2006) 